\title{
TU/e EmonOWEN

\section{Closed-form solutions for the trajectories of charged particles in an exponentially varying magnetostatic field}

\section{Citation for published version (APA):}

van Vugt, D. C., Kamp, L. P. J., \& Huijsmans, G. T. A. (2019). Closed-form solutions for the trajectories of charged particles in an exponentially varying magnetostatic field. IEEE Transactions on Plasma Science, 47(1), 296-299. [8544029]. https://doi.org/10.1109/TPS.2018.2878459

DOI:

10.1109/TPS.2018.2878459

Document status and date:

Published: 01/01/2019

\section{Document Version:}

Accepted manuscript including changes made at the peer-review stage

\section{Please check the document version of this publication:}

- A submitted manuscript is the version of the article upon submission and before peer-review. There can be important differences between the submitted version and the official published version of record. People interested in the research are advised to contact the author for the final version of the publication, or visit the $\mathrm{DOI}$ to the publisher's website.

- The final author version and the galley proof are versions of the publication after peer review.

- The final published version features the final layout of the paper including the volume, issue and page numbers.

Link to publication

\section{General rights}

Copyright and moral rights for the publications made accessible in the public portal are retained by the authors and/or other copyright owners and it is a condition of accessing publications that users recognise and abide by the legal requirements associated with these rights.

- Users may download and print one copy of any publication from the public portal for the purpose of private study or research.

- You may not further distribute the material or use it for any profit-making activity or commercial gain

- You may freely distribute the URL identifying the publication in the public portal.

If the publication is distributed under the terms of Article 25fa of the Dutch Copyright Act, indicated by the "Taverne" license above, please follow below link for the End User Agreement:

www.tue.nl/taverne

Take down policy

If you believe that this document breaches copyright please contact us at:

openaccess@tue.nl

providing details and we will investigate your claim. 


\title{
Closed-form solutions for the trajectories of charged particles in an exponentially varying magnetostatic field
}

\author{
D.C. van Vugt ${ }^{1}$, L.P.J. Kamp ${ }^{1}$, G.T.A. Huijsmans ${ }^{1,2}$ \\ ${ }^{1}$ Eindhoven University of Technology, Eindhoven, The Netherlands \\ ${ }^{2}$ CEA, IRFM, F-13108 Saint-Paul-lez-Durance, France
}

\begin{abstract}
We present a new reference solution for charged particle motion in a strongly inhomogeneous magnetostatic field. The solution describes both bound and unbound particle motion, which can be split into three regimes, the deflection, loop-deflection and drift regime. We calculate the trajectory in terms of trigonometric and hyperbolic functions, resulting in simple analytical expressions for the particle position and $\nabla B$-drift velocity. This reference solution is useful to verify and compare the performance of kinetic and guiding-center charged particle pushers in inhomogeneous fields, by verifying the conservation of two constants of motion, as well as the exact trajectory at any time.
\end{abstract}

\section{Introduction}

Computing the motion of charged particles is commonplace in research into fusion and industrial plasmas, beam and accelerator physics, molecular dynamics and astrophysics. Different approaches can be taken to numerically integrate the equation of motion, depending on the nature of the fields and trajectories. Guidingcenter methods, which treat the particle as a ring of current, can be very efficient in strongly magnetized plasmas. In other cases the full trajectory can be computed, using either implicit or explicit methods.

The most common integration method is the Boris-Buneman leapfrog method [1]-[3], which has the advantages of being explicit, secondorder and energy-conserving. Recently many new integration methods have been proposed, such as those based on splitting schemes [4], generating functions [5], or spectral deferred corrections [6]. These new methods are generally high-order and energy-conserving.

The performance of these so-called pushers or movers varies widely, depending on the fields and particle paths. For instance Runge-Kutta methods are known to have poor energy conservation properties, and some splitting methods are known to perform poorly in inhomogeneous fields [4]. To assess the performance of particle pushers they are often benchmarked using several different fields for which the exact trajectory is known analytically. Currently, the most common testcases found in literature are a simple uniform magnetic field [7]-[10] and the Penning Trap [4], [6], [11], [12]. However, these both have a uniform magnetic field, so the performance of pushers with respect to magnetic field gradients and associated drifts is not tested thoroughly. Only few closed-form analytical solutions of the equation of motion for a charged particle in non-uniform magnetic fields are known. For the case of a unidirectional magnetostatic field the path of a particle can be found but generally not in closed form. Elementary analytic results have been documented only for a few simple cases [13].

We propose a magnetic field tailor-made for testing the performance of pushers in inhomogeneous fields, with $\mathbf{B}=B_{0} e^{x / l} \hat{z}$, where $l$ is the gradient length. This magnetostatic field is inhomogeneous, simple and allows for an exact solution in closed form for the trajectories for arbitrary values of the gradient length $l$ making it ideal for benchmarking particle pushers. This field has been used before, however, without an exact solution [14]. Although inhomogeneous fields have been proposed and used before in testing particle pushers [4], [7], [10], [15], [16], we believe that this field is the only one with a simple analytical solution for the particle positions and its $\nabla \mathbf{B}$ drift at any time and for arbitrary strength of the inhomogeneity.

\section{Solution of the equation of motion}

Consider a particle with charge $q$ and mass $m$, moving in the $x-y$-plane of a Cartesian coordinate system and under the influence of 
a magnetostatic field in the $z$-direction with strength

$$
B_{z}(x)=B_{0} e^{x / l} .
$$

There is no electric field. Since the kinetic energy of the particle cannot be altered by the magnetic field we can write the velocity vector as $\mathbf{v}(\theta)=v_{\perp}(\cos (\theta) \hat{x}+\sin (\theta) \hat{y})$, using polar coordinates in velocity space. Since $v_{\perp}$ is a constant, the particle's velocity is uniquely described by $\theta(t)$. We now introduce dimensionless quantities for these variables according to

$$
\tilde{\mathbf{v}}=\mathbf{v} / v_{\perp}, \quad \tilde{x}=x / l, \quad \tilde{y}=y / l, \quad \tilde{t}=v_{\perp} t / l
$$

and drop the tildes immediately. We thus have

$$
\dot{x}=\cos \theta, \quad \dot{y}=\sin \theta,
$$

where the dot indicates a time-derivative. The time-evolution of the velocity is determined by the Lorentz force $\mathbf{F}=q \mathbf{v} \times \mathbf{B}$, which reduces to

$$
\dot{\mathbf{v}}=\alpha e^{x} \mathbf{v} \times \hat{z} .
$$

The dimensionless parameter $\alpha$ is given by

$$
\alpha=\frac{q B_{0} l}{m v_{\perp}},
$$

which is the ratio of the gradient length $l$ of the magnetic field and the gyroradius $r_{g}=$ $m v_{\perp} /\left(q B_{0}\right)$. Writing out the components $\ddot{x}$ and $\ddot{y}$ of (4) hints at an equation of motion for $\dot{\theta}$

$$
\begin{aligned}
& \ddot{x}=\quad-\dot{\theta} \sin \theta=-\dot{\theta} \dot{y}=\alpha e^{x} \dot{y} \\
& \ddot{y}=\quad \dot{\theta} \cos \theta=\quad \dot{\theta} \dot{x}=-\alpha e^{x} \dot{x}
\end{aligned}
$$

which is given by

$$
\dot{\theta}=-\alpha e^{x} .
$$

To solve (8) we can multiply both sides with $\cos \theta=\dot{x}$

$$
\dot{\theta} \cos \theta=-\alpha e^{x} \dot{x} .
$$

This can be integrated from 0 to $t$ and simplified with (8)

$$
\sin \theta-\sin \theta_{0}=\dot{\theta}-\dot{\theta}_{0} .
$$

We can further simplify and rearrange to obtain

$$
\dot{\theta}=\sin \theta-p,
$$

where the constant $p=\dot{y}+\alpha e^{x}=\sin \left(\theta_{0}\right)-\dot{\theta}_{0}$ is the dimensionless $y$-component of the generalized momentum ${ }^{1}, \theta_{0}=\theta(t=0)$ and $\dot{\theta}_{0}$ denotes the time-derivative of $\theta$ at $t=0$.

We can define an effective potential for the

\footnotetext{
${ }^{1}$ The other conserved quantity is the particle velocity
}

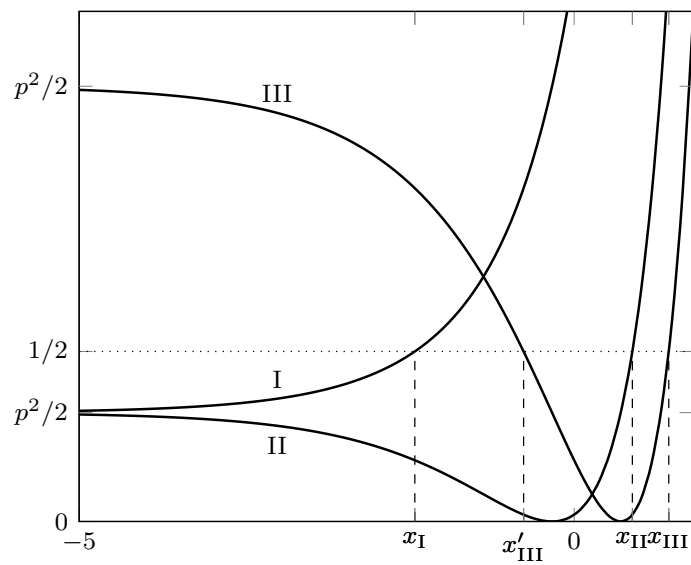

Figure 1. Effective potential $V(x)$ plotted for $\alpha=1$ in the three different regimes, with $p_{i}=-0.8,0.8,1.6 . x_{i}=$ $\ln \left(p_{i}+1\right)$ are the maximum $x$-values. The trajectories are along the line $E=1 / 2 . x_{\text {III }}^{\prime}=\ln \left(p_{\text {III }}-1\right)$ is the minimum $x$-value in the drift regime.

motion in the $x$-direction

$$
\ddot{x}=-\frac{\mathrm{d} V}{\mathrm{~d} x},
$$

which we can derive from $\dot{x}=\cos \theta$ and (8)

$$
\ddot{x}=-\dot{\theta} \sin \theta=\alpha e^{x} \dot{y}=\alpha e^{x}\left(p-\alpha e^{x}\right) .
$$

Integrating this to $x$ to obtain $V$ is straightforward, leading to

$$
V(x)=\frac{1}{2}\left(p-\alpha e^{x}\right)^{2},
$$

choosing the additive constant to be zero. Multiplying (12) with $\dot{x}$ and integrating over time leads to an expression for conservation of energy

$$
E=\frac{1}{2} \dot{x}^{2}+V(x)=\frac{1}{2} .
$$

The topology of the trajectories can now be evaluated from the effective potential $V(x)$. There are three different regimes, depending on the value of $p$ and the sign of $\alpha^{2}$. If $-1<p \leq 0$ the particle is in the deflection regime (I). The particle will approach a region of higher magnetic fields and is then being deflected. For $0<p \leq 1$ the particle is in the loopdeflection regime (II). Again the particle is moving towards the high-field region and then being deflected making a single loop. If $p>1$ the particle is in the drift or gyration regime (III). The special case $p=-1$ describes motion along the $y$-axis, with $\theta=-\frac{\pi}{2}$. If $p=0$ the motion resembles the $\supset$ symbol, with $\theta$ decreasing from 0 to $-\pi \cdot p=1$ is the transition point between loop-deflection and gyration, where the particle

\footnotetext{
${ }^{2}$ for $\alpha<0$ set $p \rightarrow-p$. We will assume $\alpha=1$ in the following.
} 
makes a single loop, starting at $\theta=\pi / 2$ and ending at $\theta=-3 \pi / 2$. Figure 1 shows the effective potential $V(x)$ in each of the regimes. The location of the turning points $x_{i}=\ln \left(p_{i} \pm 1\right)$ $(i=1,2,3)$, where $V(x)=E=1 / 2$ is also indicated.

To quantitatively describe the particle trajectories we need to solve for $\theta=\theta(t), x=x(t)$ and $y=y(t)$. We can integrate the equation of motion for $\theta,(11)$

$$
\int_{0}^{t} \mathrm{~d} t^{\prime}=\int_{\theta_{0}}^{\theta} \frac{\mathrm{d} \theta^{\prime}}{\sin \theta^{\prime}-p} .
$$

Without loss of generality we choose the initial position of the particle to be given by $x(t=0)=$ $\ln (p+1)$, which corresponds to the location of the turning point and $y(t=0)=0$. Then $\theta_{0}=$ $\pm \frac{\pi}{2}$. We will take $\theta_{0}=-\frac{\pi}{2}$ in what follows The integrals in (16) can be evaluated to obtain $t=t(\theta)$, which may then be inverted to yield:

$$
\begin{aligned}
\theta(t)= & -2 \arctan \left[\sqrt{\frac{1+p}{1-p}} \tanh \left(\frac{1}{2} \gamma t\right)\right] \\
& -\frac{\pi}{2}
\end{aligned}
$$

for $-1 \leq p<1$ and

$$
\begin{aligned}
\theta(t)= & -2 \arctan \left[\sqrt{\frac{p+1}{p-1}} \tan \left(\frac{1}{2} \gamma t\right)\right] \\
& -2 \pi\left\lfloor\frac{\gamma t}{2 \pi}\right\rceil-\frac{\pi}{2}
\end{aligned}
$$

for $p>1$. Here $\gamma=\sqrt{\left|p^{2}-1\right|}$ and $\lfloor x\rceil$ indicates the integer closest to $x$. The second term of the right-hand side of (18) is needed to select from the multiple solutions of $t=t(\theta)$ whenever $p \geq 1$.

The $x$-trajectory can be found from (8) and (11)

$$
x(t)=\ln (-\dot{\theta})=\ln (-\sin \theta(t)+p),
$$

whereas the $y$-trajectory can be found by integration of $\dot{y}=p-\alpha e^{x}=p+\dot{\theta}(t)$,

$$
y(t)=p t+\theta(t)+\frac{\pi}{2} .
$$

Figure 2 shows example particle trajectories in each of the three regimes.

The magnetic field gradient drift velocity in regime III is given by

$$
v_{d}=\langle\dot{y}\rangle=p-\gamma
$$

where $\langle\dot{y}\rangle$ denotes the time-averaged velocity in the $y$-direction. in the limit of $p \gg 1$ (and thus $p \approx \alpha$ ) we can expand to first order:

$$
v_{d}^{*}=v_{\perp}\left(p-\sqrt{p^{2}-1}\right)=\frac{v_{\perp}}{2 p}+\mathcal{O}\left(p^{-3}\right),
$$

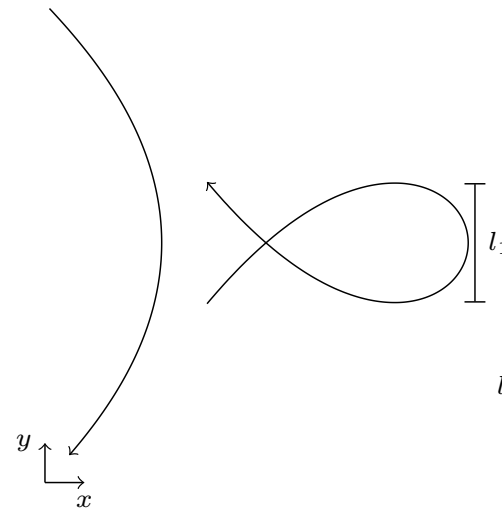

(I)

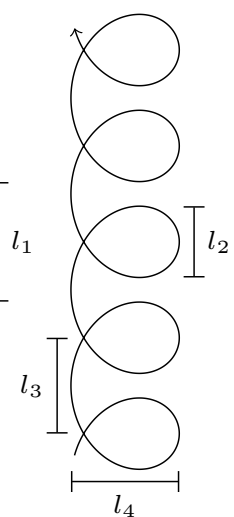

(III)
Figure 2. Paths of a charged particle in the deflection (I, $p=-0.6$ ), loop-deflection (II, $p=0.9$ ) and drift regimes (III, $p=2$ ). Indicated in the figure are $l_{1}=$ $\pi-\frac{4 p}{\gamma} \tanh ^{-1}\left(\sqrt{\frac{1-p}{1+p}}\right), l_{2}=\pi-\frac{4 p}{\gamma} \tan ^{-1}\left(\sqrt{\frac{p-1}{p+1}}\right)$, $l_{3}=\frac{2 \pi}{\gamma}(p-\gamma), l_{4}=\ln \left(\frac{p+1}{p-1}\right)$, where $\gamma=\sqrt{\left|p^{2}-1\right|}$.

which reduces to the well-known drift expression for small magnetic field gradients $[17$, p. 74$]$

$$
\mathbf{v}_{d} \approx-\frac{m v_{\perp}^{2}}{2 q B} \frac{(\nabla B) \times \mathbf{B}}{B^{2}}=\frac{m v_{\perp}^{2}}{2 q|\mathbf{B}|} \frac{1}{l} \hat{y}=\frac{v_{\perp}}{2 \alpha} \hat{y}
$$

where $B=|\mathbf{B}|$ and $\hat{e}_{y}$ is the unit vector in the $y$-direction.

\section{Verification}

To show an example application and test the correctness of the solution we trace a set of protons with mass $m=1.67262158 \cdot 10^{-27} \mathrm{~kg}$ with the Boris-Buneman method [1]-[3] with timestep $\Delta t=2 \mathrm{~ns}$. The magnetic field has a strength of $B_{0}=1 \mathrm{~T}$ and a gradient length $l=0.2 \mathrm{~mm}$. Cases studied are the same as in figure 2, i.e. $p=-0.6, p=0.9$ and $p=2$. Particles are initialized at $\left(x\left(t_{0}\right), y\left(t_{0}\right)\right)$ with $t_{0}=$ $-30,-40,-91 \mathrm{~ns}$, where $x(t)$ and $y(t)$ are given by (19) and (20) respectively. The particle paths are then followed until $t_{1}=30,40,91 \mathrm{~ns}$ respectively. Figure 3 shows a comparison between the exact particle trajectories and a numerical calculation. The final error in the numerical trajectory calculation, $\epsilon=\left|\mathbf{x}_{\text {num }}\left(t_{1}\right)-\mathbf{x}\left(t_{1}\right)\right|_{2}$, scales with the timestep size and is shown in figure 4 .

\section{Conclusion}

We present a new and simple solution for the trajectories of a charged particle in an exponentially varying, inhomogeneous magnetostatic field having a constant field gradient of arbitrary magnitude. Three regimes are identified, 


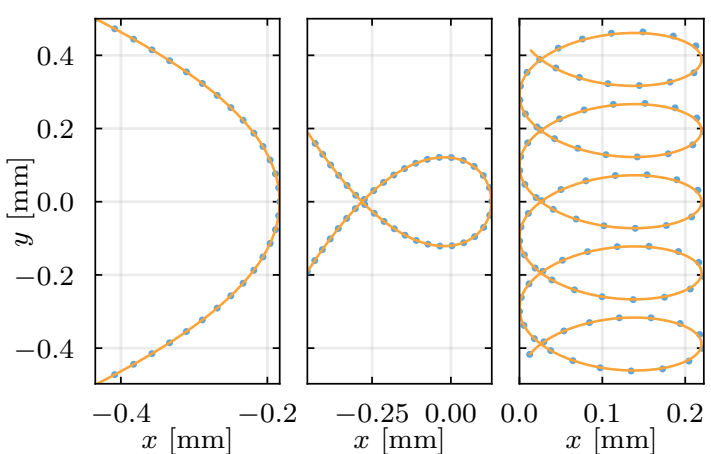

Figure 3. Comparison of particle paths with numerically integrated trajectories in regime (I), (II) and (III) from left to right. The solid line indicates the exact solution, the dots indicate the particle position as calculated with the Boris-Buneman method.

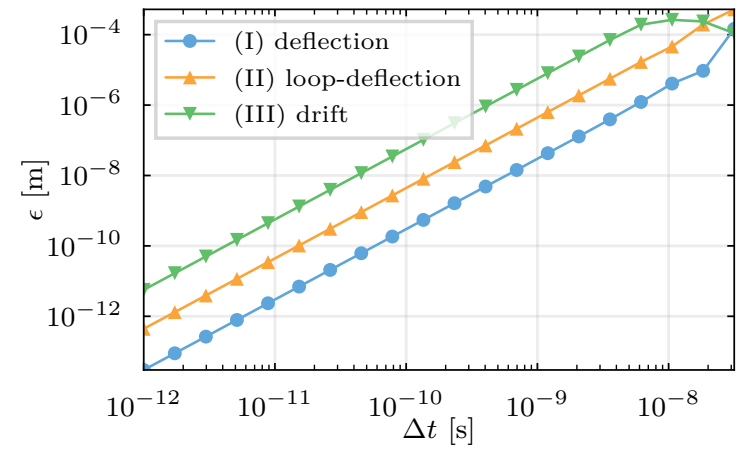

Figure 4. Distance between endpoints of paths in figure 3 versus timestep size.

depending on the value of the conserved component of the generalized momentum. These are the so-called deflection, loop-deflection, and drift regimes. The drift velocity in the last regime reduces to the standard expression in the limit of small field gradients. Closed-form analytical solutions are presented for the motion of the particle in each of these regimes and compared against numerical calculations. This magnetic field is well-suited for testing particle pushers even in strongly inhomogeneous fields. The performance of kinetic and guiding-center methods can be evaluated through comparison with the presented analytical expressions for the particle trajectory, drift velocity and conserved quantities.

\section{Acknowledgements}

This work is part of the research programme 09KE02-1, which is (partly) financed by the Netherlands Organisation for Scientific Research (NWO).

\section{References}

[1] J. P. Boris, "Relativistic plasma simulationoptimization of a hybrid code," in Proc. Fourth Conf. Num. Sim. Plasmas, 1970, pp. 3-67.

[2] C. K. Birdsall and A. B. Langdon, Plasma Physics via Computer Simulation, ser. Series in Plasma Physics. Taylor \& Francis, 2004.

[3] O. Buneman, "Time-reversible difference procedures," J. Comput. Phys., vol. 1, no. 4, pp. 517-535, 1967.

[4] C. Knapp, A. Kendl, A. Koskela, and A. Ostermann, "Splitting methods for time integration of trajectories in combined electric and magnetic fields," Phys. Rev. E, vol. 92, no. 6, p. 063310, 2015.

[5] R. Zhang, H. Qin, Y. Tang, J. Liu, Y. He, and J. Xiao, "Explicit symplectic algorithms based on generating functions for charged particle dynamics," Phys. Rev. E - Stat. Nonlinear, Soft Matter Phys., vol. 94, no. 1, pp. 1-12, 2016.

[6] M. Winkel, R. Speck, and D. Ruprecht, "A highorder Boris integrator," J. Comput. Phys., vol. 295, pp. $456-474,2015$.

[7] S. A. Chin, "Symplectic and energy-conserving algorithms for solving magnetic field trajectories," Phys. Rev. E - Stat. Nonlinear, Soft Matter Phys., vol. 77 , no. 6 , pp. $1-12,2008$.

[8] V. Fuchs and J. P. Gunn, "On the integration of equations of motion for particle-in-cell codes," J. Comput. Phys., vol. 214, no. 1, pp. 299-315, 2006.

[9] Y. He, Y. Sun, J. Liu, and H. Qin, "Higher order volume-preserving schemes for charged particle dynamics," J. Comput. Phys., vol. 305, pp. 172-184, 2016.

[10] H.-s. Mao and R. E. Wirz, "Comparison of Charged Particle Tracking Methods for Non-Uniform Magnetic Fields," 42nd AIAA Plasmadynamics Lasers Conf., no. June, pp. AIAA 2011-3739, 2011.

[11] Y. He, Y. Sun, R. Zhang, Y. Wang, J. Liu, and H. Qin, "High order volume-preserving algorithms for relativistic charged particles in general electromagnetic fields," Phys. Plasmas, vol. 23, no. 9, p. 092109, 2016.

[12] L. Patacchini and I. H. Hutchinson, "Explicit timereversible orbit integration in Particle In Cell codes with static homogeneous magnetic field," J. Comput. Phys., vol. 228, no. 7, pp. 2604-2615, 2009.

[13] P. C. Clemmow and J. P. Dougherty, Electrodynamics of particles and plasmas. Addison-Wesley, 1969.

[14] H. X. Vu and J. U. Brackbill, "Accurate Numerical Solution of Charged Particle Motion in a Magnetic Field," p. 384, 1995.

[15] T. C. Genoni, R. E. Clark, and D. R. Welch, "A Fast Implicit Algorithm for Highly Magnetized Charged Particle Motion," Open Plasma Phys. J., vol. 3, no. 2, pp. 36-41, 2010.

[16] H. Qin, S. Zhang, J. Xiao, J. Liu, Y. Sun, and W. M. Tang, "Why is Boris algorithm so good?" Phys. Plasmas, vol. 20, no. 8, p. 084503, 2013.

[17] J. A. Bittencourt, Fundamentals of plasma physics. Springer Science \& Business Media, 2013. 